\title{
Gastrodin inhibits high glucose-induced human retinal endothelial cell apoptosis by regulating the SIRT1/TLR4/NF-кBp65 signaling pathway
}

\author{
TONG-HE ZHANG ${ }^{1}$, CHUN-MEI HUANG $^{1}$, XUE GAO $^{2}$, JIA-WEI WANG $^{2}$, LIN-LIN HAO $^{2}$ and QIANG JI ${ }^{1}$ \\ ${ }^{1}$ Department of Ophthalmology, The Second People's Hospital of Jinan, Jinan, Shandong 250001; \\ ${ }^{2}$ Department of Ophthalmology, The Second Hospital of Shandong University, Jinan, Shandong 250031, P.R. China
}

Received December 25, 2017; Accepted March 6, 2018

DOI: $10.3892 / \mathrm{mmr} .2018 .8841$

\begin{abstract}
Diabetic retinopathy (DR), one of the most common complications of late-phase diabetes, is associated with the ectopic apoptosis of microvascular cells. Gastrodin, a phenolic glucoside derived from Gastrodia elata Blume, has been reported to have antioxidant and anti-inflammation activities. The aim of the present study was to investigate the effects of gastrodin on high glucose (HG)-induced human retinal endothelial cell (HREC) injury and its underlying mechanism. The results demonstrated that $\mathrm{HG}$ induced cell apoptosis in HRECs, which was accompanied by increased levels of reactive oxygen species production. Gastrodin treatment significantly alleviated $\mathrm{HG}$-induced apoptosis and oxidative stress. Furthermore, HG stimulation decreased the levels of SIRT1, which was accompanied by an increase in Toll-like receptor 4 (TLR4) expression and the levels of phosphorylated nuclear factor (NF)- $\mathrm{Bp} 65$. However, the administration of gastrodin significantly inhibited the activation of the sirtuin 1 (SIRT1)/TLR4/NF-кBp65 signaling pathway in HRECs exposed to HG. Collectively, the present study demonstrated that gastrodin may be effective against HG-induced apoptosis and its action may be exerted through the regulation of the SIRT1/TLR4/NF-кBp65 signaling pathway.
\end{abstract}

\section{Introduction}

Diabetes is a major public health problem affecting 415 million people worldwide and diabetic retinopathy (DR) is a

Correspondence to: Dr Qiang Ji, Department of Ophthalmology, The Second People's Hospital of Jinan, 148 Jingyi Road, Jinan, Shandong 250001, P.R. China

E-mail: jiqiang_063@163.com

Abbreviations: DR, diabetic retinopathy; HG, high glucose; HRECs, human retinal endothelial cells; ROS, reactive oxygen species; TLR4, Toll-like receptor 4; NF- $\kappa \mathrm{B}$, nuclear factor- $\kappa \mathrm{B}$

Key words: diabetic retinopathy, high glucose, human retinal endothelial cells, gastrodin, apoptosis major diabetic complication that can cause significant visual impairment and blindness (1). The early stages of DR can be inhibited by improvement of glycemic control suggesting hyperglycemia to initiate the pathology of the pathology of DR $(2,3)$. Emerging evidence showed that human retinal endothelial cells (HRECs) dysfunction is the initial event of microvascular disorder in the development of DR. HRECs damage is associated with thickening of the capillary endothelial basement membrane (4). Moreover, current studies demonstrated that except sterile-inflammation, high glucose $(\mathrm{HG})$ induced oxidative stress in the retina also plays a critical role in the pathogenesis of retinopathy (5). Administration of antioxidants or genetic overexpression of superoxide dismutase has been demonstrated to inhibit the diabetes-induced degeneration of retinal capillaries (6-8). Accordingly, strategies against intracellular reactive oxygen species (ROS) production induced cell apoptosis help to reduce HRECs injury (9).

Gastrodin, a major active ingredient of Chinese herbal medicine called Gastrodia elata Blume, has been reported to exert anti-inflammatory and anti-apoptotic effects in various diseases $(10,11)$. Importantly, Peng et al (12) found gastrodin treatment reduced reactive oxygen species production in macrophages and protected macrophages against oxidative stress-induced apoptosis. And a recent study identified that gastrodin could stimulate M2 macrophage polarization and rescue macrophages from oxidative stress-induced apoptosis and death (13). To date, no literatures involved in the role of gastrodin on HG-induced HRECs injury.

In the present study, we sought to examine the potential protective effects of gastrodin on $\mathrm{HG}$-induced HRECs damage and to investigate the relationship between its effect and the modulation of TLR4/NF- $\mathrm{Bp} 65$ signaling pathway. And our work suggested that gastrodin may have the potential to be a novel pharmaceutical approach for the treatment of DR.

\section{Materials and methods}

Cell culture. Human retinal endothelial cells (HRECs) were obtained from Cell Systems (Kirkland, WA, USA). The culture medium was EGM-2 Bulletkit medium (Lonza, 
Basel Switzerland) with $100 \mathrm{U} / \mathrm{ml}$ penicillin/100 $\mu \mathrm{g} / \mathrm{ml}$ streptomycin (Invitrogen; Thermo Fisher Scientific, Inc., Waltham, MA, USA), containing $10 \%$ fetal bovine serum (Gibco; Thermo Fisher Scientific, Inc.). Cells were maintained at $37^{\circ} \mathrm{C}$ and $5 \% \mathrm{CO}_{2}$. HRECs of passages 6-10 were used for experiments.

Cell treatment. After an initial $24 \mathrm{~h}$ of culture in serum-free medium, HRECs were treatment with various concentration of gastrodin $(0.1,1,10,100 \mu \mathrm{M})$ or $100 \mathrm{nM}$ gastrodin in normal medium for various time $(6,12,24$ and $48 \mathrm{~h})$ to detect the optimal concentration and time of intervention. Cells were subjected to medium containing $5 \mathrm{mM}$ glucose (control group) or high glucose medium containing $30 \mathrm{mM}$ glucose (HG group). HRECs cultured in 6-well plates were transfected with recombinant plasmid pcDNA3.1 or pcDNA3.1-SIRT1 (Invitrogen; Thermo Fisher Scientific, Inc.) to overexpress SIRT1 using Lipofectamine 2000 (Invitrogen; Thermo Fisher Scientific, Inc.) in accordance with the manufacturer's protocol.

Cell viability assay. The Cell Counting Kit-8 (CCK-8, Biosharp, Hefei, China) was performed to determine cell viability of HRECs. In brief, $5 \times 10^{3}$ of cells were cultured in a 96-well plate at $37^{\circ} \mathrm{C}$ and allowed to attach for $24 \mathrm{~h}$. After starvation for $12 \mathrm{~h}$, cells were stimulated with different concentrations of gastrodin for different time in normal median. Then, $10 \mu 1$ CCK-8 solution was added to each well, plates were incubated at $37^{\circ} \mathrm{C}$ for another $2 \mathrm{~h}$. The absorbance of cells was then measured at $450 \mathrm{~nm}$.

Intracellular reactive oxygen species (ROS) analysis. The intracellular ROS level was measured by dihydroethidium (DHE; Beyotime Institute of Biotechnology, Haimen, China) staining. After various treatment, HRECs were incubated with $5 \mu \mathrm{M} \mathrm{DHE}$ at $37^{\circ} \mathrm{C}$ for $30 \mathrm{~min}$. Following incubation, cells were fed with normal growth medium without DHE for $1 \mathrm{~h}$, and rinsed with PBS. Fluorescence images were observed using a fluorescence microscope (Carl Zeiss AG, Oberkochen, Germany). The fluorescence intensity was measured using ImageJ software (NIH, Bethesda, MD, USA).

Reverse transcription-quantitative polymerase chain reaction $(R T-q P C R)$. RT-qPCR analyses were carried out as described previously (14). Total RNA was extracted from cells in groups using TRIzol reagent (Invitrogen; Thermo Fisher Scientific, Inc.) and quantified by measuring the absorbance at $260 \mathrm{~nm}$. Subsequently, $2 \mu \mathrm{l}$ of total RNA was used for the preparation of cDNA by reverse transcription using the PrimeScript RT reagent kit (Takara Bio, Inc., Otsu, Japan) according to the manufacturer's instructions. The expression of HO-1, NQO1, NRF2 and GCLM mRNA were determined on the Applied Biosystems StepOne Real-Time PCR system (Applied Biosystems; Thermo Fisher Scientific, Inc.). The reaction condition was $95^{\circ} \mathrm{C}$ at $10 \mathrm{~min}$ for a hot start, then 40 cycles at $95^{\circ} \mathrm{C}$ for $15 \mathrm{sec}, 60^{\circ} \mathrm{C}$ for $60 \mathrm{sec}$ and $72^{\circ} \mathrm{C}$ for $60 \mathrm{sec}$. The primer sequences used in this study were listed in Table I. The gene expression level was calculated by $2^{-\Delta \Delta \mathrm{Cq}}$ methods and normalized to 18S RNA. All experiments were repeated in three times.
Table I. Forward and reverse primers used for reverse transcription-quantitative polymerase chain reaction.

\begin{tabular}{|c|c|c|}
\hline Gene name & Direction & Sequence $\left(5^{\prime}-3^{\prime}\right)$ \\
\hline \multirow[t]{2}{*}{$\mathrm{HO}-1$} & Forward & GGCAGAGGGTGATAGAAGAGG \\
\hline & Reverse & AGCTCCTGCAACTCCTCAAA \\
\hline \multirow[t]{2}{*}{ NQO1 } & Forward & TCCAGAAACGACATC \\
\hline & Reverse & GCACCCCAAACCAATACAAT \\
\hline \multirow[t]{2}{*}{ NRF2 } & Forward & AAGAATAAAGTCGCCGCCCA \\
\hline & Reverse & AGATACAAGGTGCTGAGCCG \\
\hline \multirow[t]{2}{*}{ GCLM } & Forward & AGTCTCCATGGAAGAACGGCC \\
\hline & Reverse & CGATTACGGCTTCACTTGCCT \\
\hline \multirow[t]{2}{*}{$18 \mathrm{~S}$} & Forward & GAGGGGAGAGCGGGTAAGA \\
\hline & Reverse & TCGGGGTCCGACAAAACCC \\
\hline
\end{tabular}

NRF2, nuclear factor-E2-related factor 2; HO-1, hemeoxygenase-1; NQO1, nicotinamide adenine dinucleotide phosphate dehydrogenase quinone 1; GCLM, $\gamma$-glutamate-cysteine ligase modifier.

Cell apoptosis assay. HRECs cell apoptosis rates were measured by flow cytometric analysis using Annexin V-FITC-PI Apoptosis Detection kit (Vazyme Biotech, Nanjing, China). Briefly, cells were trypsinized after treatment and rinsed with PBS to achieve the final concentration of $5 \times 10^{3} / \mathrm{ml}$. Then, $195 \mu \mathrm{l}$ cell suspension and $5 \mu \mathrm{l}$ Annexin V-FITC were mixed under dark for 15 min followed by stained with $10 \mu \mathrm{l}$ of propidium iodide (PI) for another $5 \mathrm{~min}$. The apoptosis rate was assayed by flow cytometry (FACSCalibur; Bio-Rad Laboratories, Inc., Hercules, CA, USA).

Western blot analysis. After treatment, HRECs lysates were collected in RIPA lysis buffer supplemented with protease/phosphatase inhibitor cocktail (Merck KGaA, Darmstadt, Germany) and total protein concentrations were measured using the BCA assay (Beyotime Institute of Biotechnology). Protein samples were loaded on 6-12\% SDS-PAGE, transferred to polyvinylidene fluoride membranes (Bio-Rad) and were blocked with $5 \%$ skim milk for $1 \mathrm{~h}$ at room temperature. The membranes were incubated with the corresponding primary antibodies against SIRT1 (1:400; Santa Cruz Biotechnology, Inc., Dallas, TX, USA), Bcl-2 (1:1,000), Bax (1:1,000), cleaved caspase 3 (1:400), cytochrome C (1:1,000), TLR4 (1:500), NF-кBp65

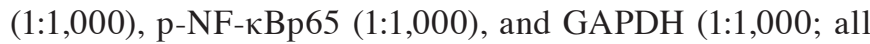
from Cell Signaling Technology, Inc., Danvers, MA, USA) at $4^{\circ} \mathrm{C}$ overnight. Subsequently, the blots were washed with PBST and incubated with a peroxidase conjugated immunoglobulin G secondary antibody (Santa Cruz Biotechnology, Inc.) for $1 \mathrm{~h}$ at room temperature. Signals were visualized using an enhanced chemiluminescence kit (GE Healthcare, Chicago, IL, USA).

Statistical analysis. All data are expressed as the mean \pm standard deviation, and analyzed with GraphPad Prism 5 (GraphPad Software, Inc., La Jolla, CA, USA). Statistical significance was tested using one-way analysis of variance (ANOVA) with Tukey's post hoc test, Kruskal-Wallis test with Dunn's post hoc test and two-way ANOVA. $<$ P 0.05 
A
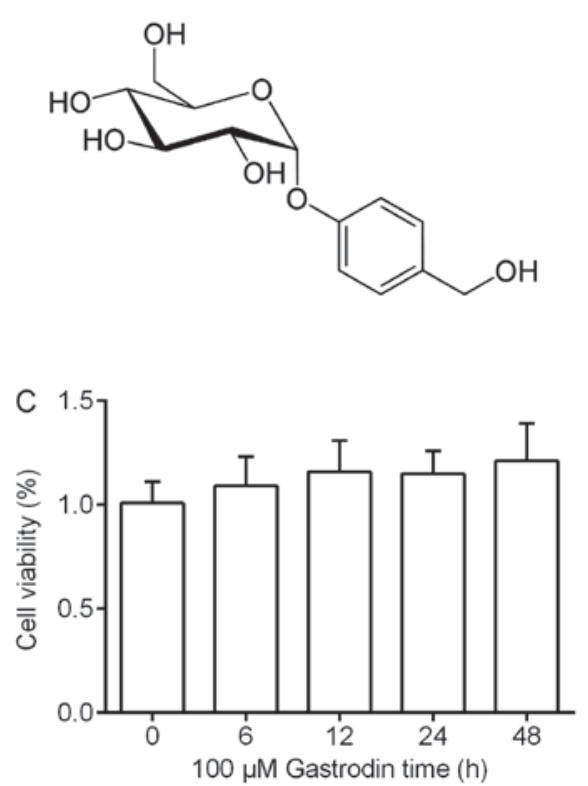
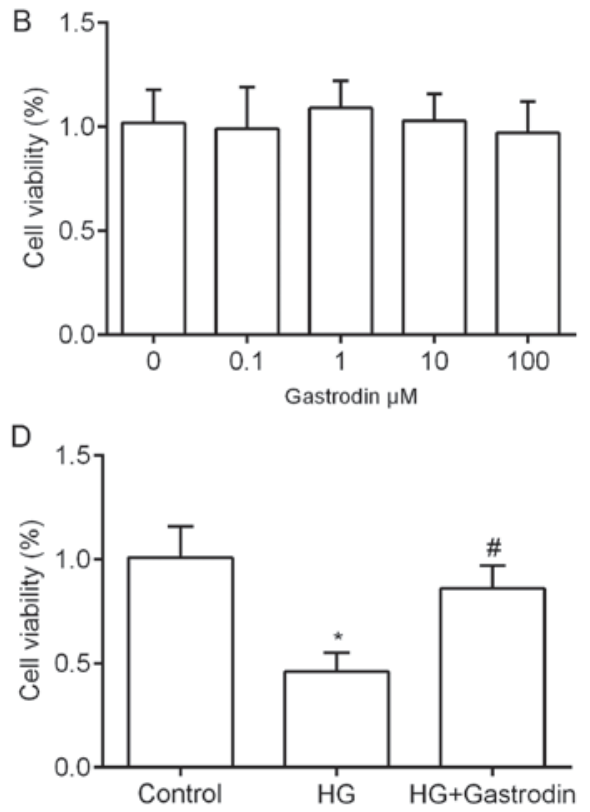

Figure 1. Gastrodin alleviates HG induced reduction in cell viability. (A) Molecular structure of gastrodin. (B) Cell Counting kit-8 assay demonstrated that gastrodin had no influence on cell viability within a range from 0.1 to $100 \mu \mathrm{M}$. (C) Treatment with $100 \mu \mathrm{M}$ gastrodin for $6,12,24$ and $48 \mathrm{~h}$ had no effect on cell viability. (D) The addition of $100 \mu \mathrm{M}$ gastrodin significantly improved the $30 \mathrm{mM}$ glucose-induced reduction of cell viability. ${ }^{*} \mathrm{P}<0.05 \mathrm{vs}$. control; ${ }^{\#} \mathrm{P}<0.05$ vs. HG. HG, high glucose.

was considered to indicate a statistically significant difference. All experiments were performed at least three times.

\section{Results}

Gastrodin ameliorated HG induced inhibition of HRECs viability. Firstly, to determine whether gastrodin (Fig. 1A) itself affects the cell viability of HRECs in normal median, cells were treated with various concentration of gastrodin. The results revealed that gastrodin had no effects on cell viability even with high concentration at $100 \mu \mathrm{M}$ (P>0.05; Fig. 1B). In addition, $100 \mu \mathrm{M}$ gastrodin had no significant effect on cell viability within $48 \mathrm{~h}$ ( $\mathrm{P}>0.05$; Fig. $1 \mathrm{C})$. Then, we found HRECs treated with HG for 24 h showed significant decrease in cell viability compared to the control group, but $100 \mu \mathrm{M}$ gastrodin markedly ameliorated the inhibitory effect caused by HG (Fig. 1D).

Gastrodin inhibited HG-induced HRECs apoptosis. HRECs apoptosis rates was measured and Fig. 2A demonstrated that, comparised with the control group, treating of HRECs with HG for $24 \mathrm{~h}$ significantly increased apoptosis, which could be reversed by addition of $100 \mu \mathrm{M}$ gastrodin (Fig. 2A). Additionally, we examined the expression levels of mitochondrial apoptotic markers by Western blot analysis. Expectedly, the ratio of $\mathrm{Bcl}-2 / \mathrm{Bax}$ was decreased, whereas the expressions of cytochrome $\mathrm{C}$ and cleaved caspase 3 were increased with the treatment of HG. When the cells were treated with both HG and $100 \mu \mathrm{M}$ gastrodin, above changes were at least partly abolished (Fig. 2B-D).

Gastrodin alleviated $H G$-induced activation of oxidative stress. Sincere the key role of oxidative stress in HG-triggered cell apoptosis, we tested the levels of ROS production in
HRECs. Intriguing, HG treatment caused increase in ROS generation compared to the control and $100 \mu \mathrm{M}$ gastrodin alleviated ROS generation significantly (Fig. 3A). Subsequently, we tested the expression levels of oxidative stress related genes including hemeoxygenase-1 (HO-1), NAD(P)H dehydrogenase quinone 1 (NQO1), NF-E2-related factor 2 (NRF2), and $\gamma$-glutamate-cysteine ligase modifier (GCLM) via RT-qPCR. $\mathrm{HG}$ dramatically increased the mRNA levels of above four genes compared to the control group. However, co-treatment with HG and gastrodin resulted in a decrease in the expression of oxidative stress related genes (Fig. 3B-E).

Gastrodin induced activation of SIRT1 and subsequent

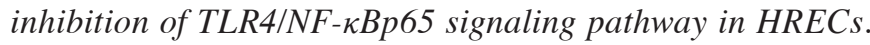
Activation of NF- $\kappa$ B was shown to promote expression of various pro-apoptotic regulators in HRECs (15) and TLR-mediated $\mathrm{NF}-\kappa \mathrm{B}$ activation were commonly shown in HRECs under HG conditions (16). We thereby investigated whether TLR4/NF- $\mathrm{Bp} 65$ signaling pathway was involved in protective of gastrodin on HRECs. As shown in Fig. 4A, HG inhibited the increase in TLR4 protein expression level and phosphorylation of $\mathrm{NF}-\kappa \mathrm{B}$ in a concentration dependent manner. Whereas, $100 \mu \mathrm{M}$ gastrodin inhibited the activation of TLR4/NF- $\kappa \mathrm{BP} 65$ induced by HG (Fig. 4B). In addition, emerging evidence suggested that SIRT1 appears to target numerous cellular factors to regulate oxidative stress and apoptosis resulting in improving diabetic retinopathy. Fig. $4 \mathrm{C}$ revealed that the expression level of SIRT1 protein was decreased in a $\mathrm{HG}$ concentration-dependent manner. However, when the HRECs were co-treated with gastrodin with HG, SIRT1 protein expression was significantly increased as compared with that treated with HG along (Fig. 4D). Moreover, overexpression of SIRT1 was then conducted to further validate the pathway involved. As a result, SIRT1 was increased by pcDNA3.1-SIRT1 but 
A

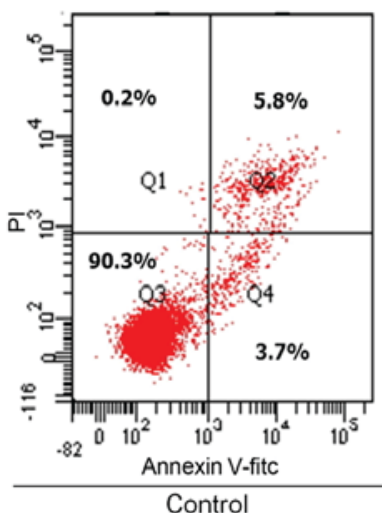

B

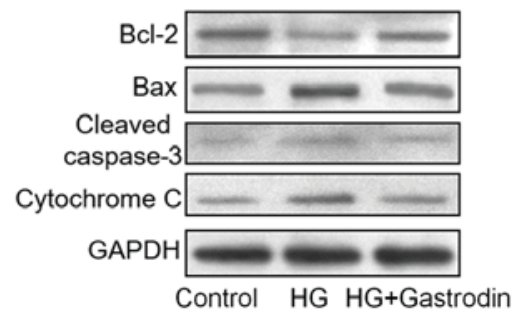

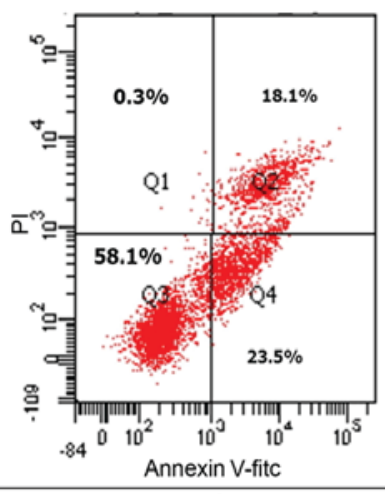

HG

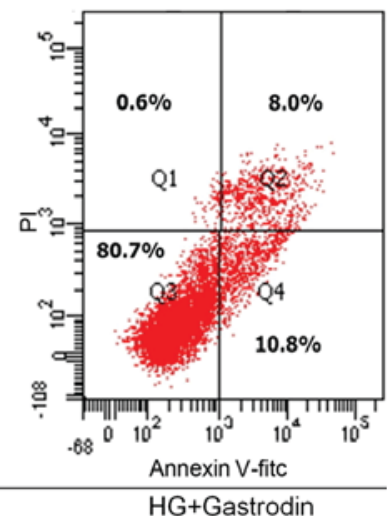

C

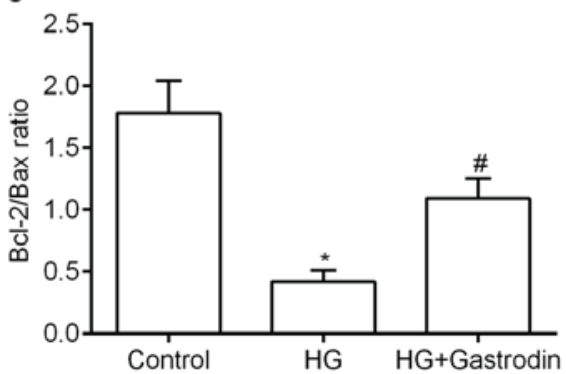

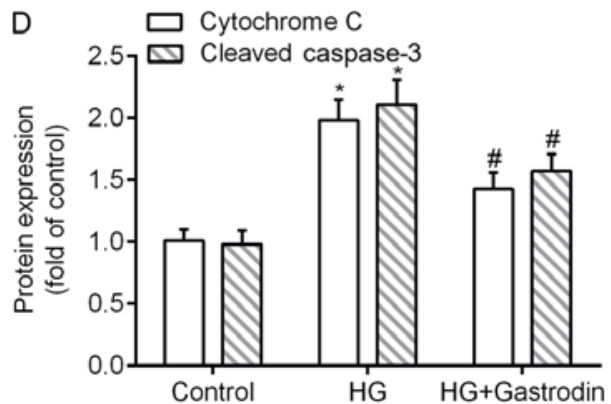

Figure 2. Gastrodin inhibits HG induced HREC apoptosis. (A) Apoptosis rate was determined by flow cytometry; the rate was expressed as the percentage of Annexin V positive plus Annexin V/PI double positive cells (Q2+Q4). (B) Western blotting demonstrated that (C) HG treatment markedly reduced the $\mathrm{Bcl}-2 / \mathrm{Bax}$ ratio; however, intervention with $100 \mu \mathrm{M}$ gastrodin increased the Bcl-2/Bax ratio in HRECs. (D) The expression levels of cytochrome $\mathrm{C}$ and cleaved caspase 3 were elevated in HG treated HRECs, and these levels reduced when cells were treated with $100 \mu \mathrm{M}$ gastrodin. ${ }^{*} \mathrm{P}<0.05$ vs. control; ${ }^{*} \mathrm{P}<0.05$ vs. HG. HG, high glucose; HRECs, human retinal endothelial cells; PI, propidium iodide; FITC, fluorescein isothiocyanate; Bcl-2, B-cell lymphoma 2; Bax, Bcl-2-associated X protein.

A

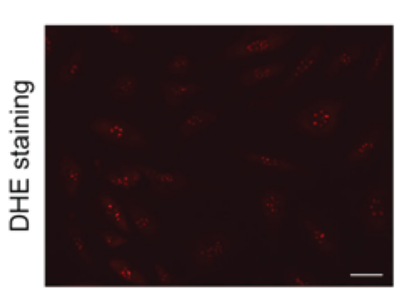

Control

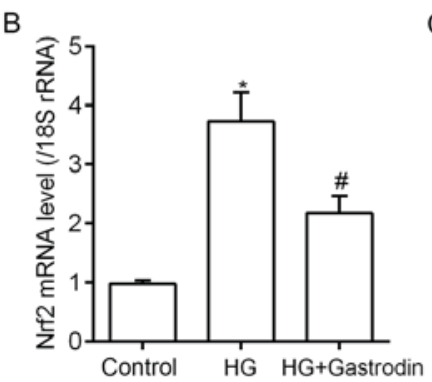

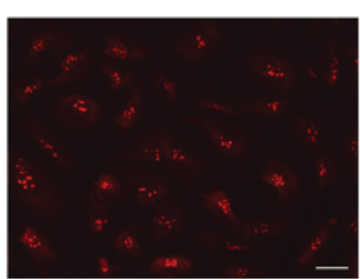

HG

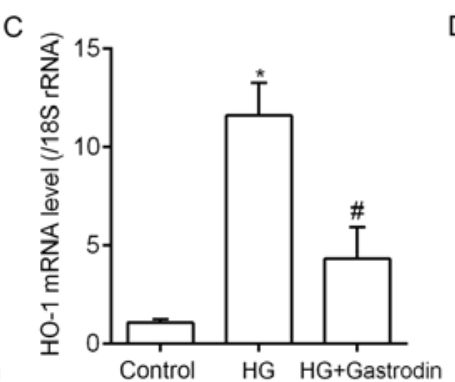

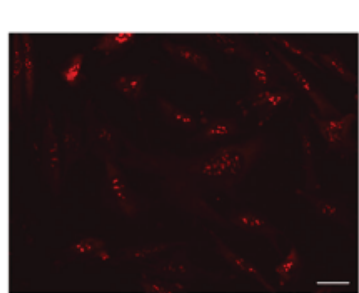

HG+Gastrodin

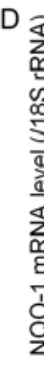

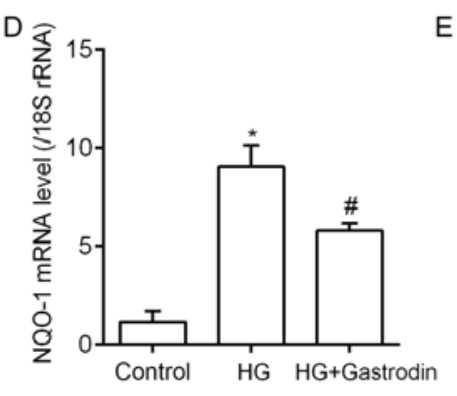

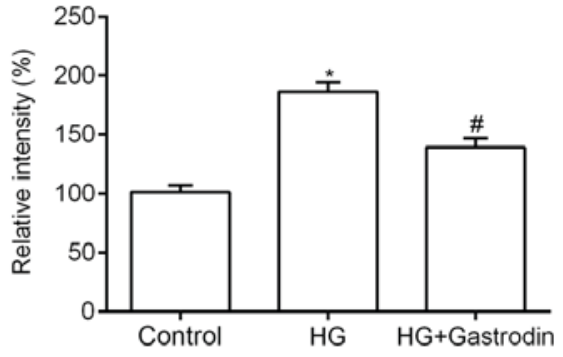

E

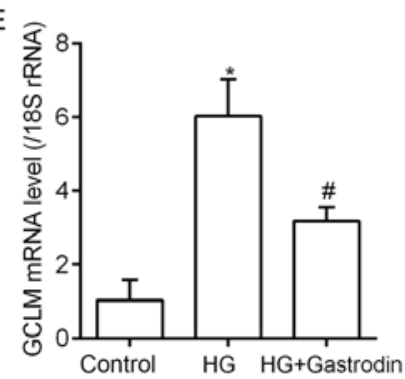

Figure 3. Gastrodin suppresses HG-induced HREC oxidative stress. (A) ROS production was detected by DHE staining and the results revealed that $100 \mu \mathrm{M}$ gastrodin significantly inhibited the increased ROS levels in HRECs induced by HG (scale bars, $50 \mu \mathrm{m}$ ). Changes in the transcription levels of the oxidative stress associated genes (B) NRF2, (C) HO-1, (D) NQO1 and (E) GCLM were detected through reverse transcription-quantitative polymerase chain reaction. The results demonstrated that $\mathrm{HG}$ induced significant gene upregulation, which gastrodin treatment attenuated. ${ }^{\mathrm{P}}<0.05$ vs. control; ${ }^{*} \mathrm{P}<0.05$ vs. HG. HG, high glucose; HRECs, human retinal endothelial cells; ROS, reactive oxygen species; DHE, dihydroethidium; NRF2, nuclear factor-E2-related factor 2; HO-1, hemeoxygenase-1; NQO1, nicotinamide adenine dinucleotide phosphate dehydrogenase quinone 1; GCLM, $\gamma$-glutamate-cysteine ligase modifier.

no significant change with HG or pcDNA3.1-vector treatment were observed (Fig. 4E). Compared with vector group with

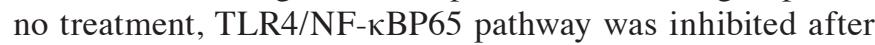

SIRT1 overexpressed. Besides, overexpression of SIRT1 at least partly reversed TLR4/NF-кBP65 activation induced by HG in HRECs in the vector group (Fig. 4F). 

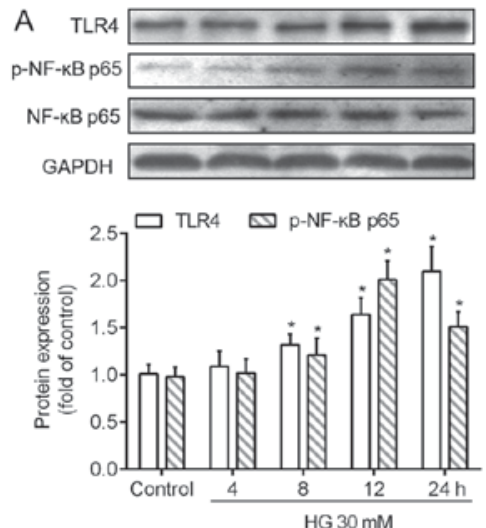

$\mathrm{D}$
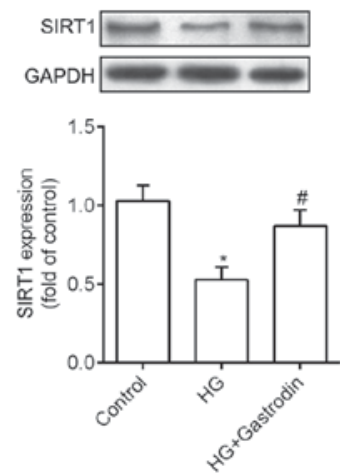

$\mathrm{B}$
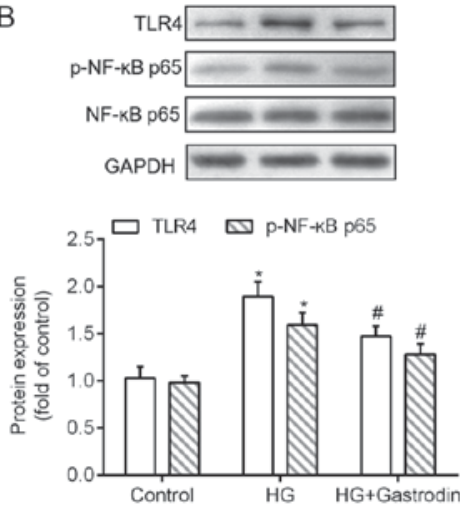

E
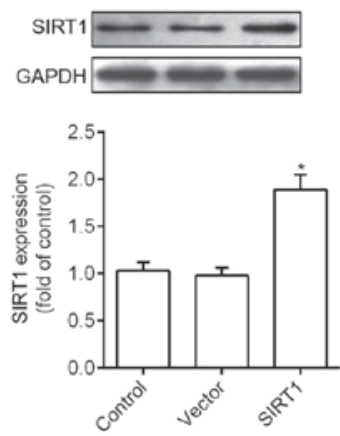

$\mathrm{C}$
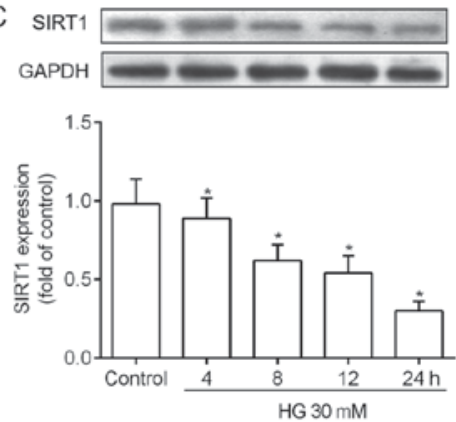

$\mathrm{F}$
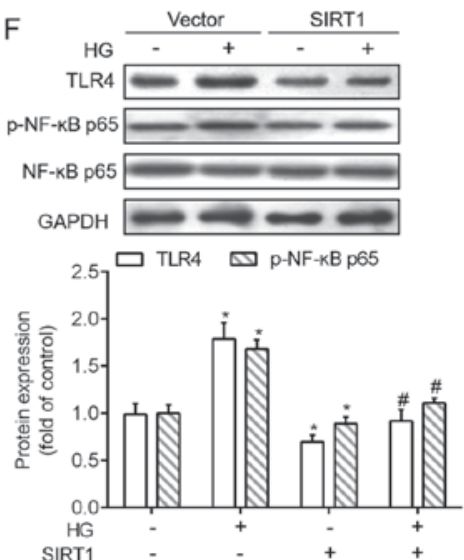

Figure 4. Gastrodin inhibits HG-induced HREC injury via the regulation of the SIRT1/TLR4/NF- $\kappa$ BP65 signaling pathway. (A) The levels of TLR4, p-NF- $\mathrm{B} / \mathrm{NF}-\kappa \mathrm{B}$ were increased in HG-stimulated HRECs in a dose-dependent manner. (B) Western blot analysis showed that gastrodin effectively inhibited the HG-induced activation of the TLR4/NF-кBp65 signaling pathway. (C) The levels of SIRT1 protein were reduced in HG-stimulated HRECs in a dose-dependent manner. (D) Gastrodin treatment reversed the HG-induced reduction in the expression of SIRT1. (E) The HRECs were transfected with pcDNA3.1-SIRT1 for $48 \mathrm{~h}$, then the protein expression of SIRT1 was detected by western blotting. (F) Overexpression of SIRT1 decreased the protein expression of TLR4 and p-NF-кBp65 in HRECs stimulated with HG. ${ }^{*} \mathrm{P}<0.05$ vs. control; ${ }^{*} \mathrm{P}<0.05$ vs. HG. HG, high glucose; HRECs, human retinal endothelial cells;

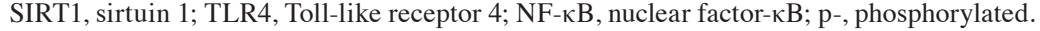

\section{Discussion}

Diabetic retinopathy (DR) is one of the main microvascular complications of diabetes mellitus and one of the leading causes of blindness worldwide (17). A large body of evidences showed that inhibition of HRECs apoptosis may have protective effects against DR $(18,19)$. In this study, we demonstrated that interference of gastrodin counteracted HG-induced oxidative stress-mediated apoptosis of HRECs.

Gastrodin, which is also known a 4-Hydroxybenzyl alcohol 4-O-beta-D-glucopyranoside, was widely used clinically as an anticonvulsant, an analgesic, and a sedative that was effective against vertigo, general paralysis, epilepsy and tetanus $(20,21)$. It is available in the market in the form of tablets, capsules and injections. Gastrodin can be absorbed sufficiently and rapidly in the intestine (22), which was facilitated by SGLT1 (23). A study identified that administration of gastrodin effectively attenuated the allodynia and hyperalgesia-related to the experimental diabetes by reciprocal regulation of sodium and potassium currents in small dorsal root ganglion neurons (24). Researchers also indicated that gastrodin could restrain the hypoxia-induced calcium ion and nitric oxide increase in cultured rat hippocampal neurons (25). Besides, gastrodin was found to decrease cell apoptosis and reduce the release of inflammatory factors (10). Similarly, the anti-apoptosis effect of gastrodin was observed in our study in HRECs stimulated by HG. In mitochondria-dependent apoptosis, the released cytochrome c activates caspase- 9 and sequentially activates the downstream effector caspase-3 (26). Antiapoptotic Bcl-2 protein inhibits the release of cytochrome $\mathrm{c}$, while proapoptotic Bax enhances the progression of apoptosis (27). Similar with previous studies $(10,28)$, we showed that HG-mediated reduction of the $\mathrm{Bcl}-2 / \mathrm{Bax}$ ratio was increased by the treatment with gastrodin in HRECs. In addition, HG-induced activation of the caspase 3 was suppressed by gastrodin. The underlying molecular mechanisms of gastrodin-mediated protection of HG-indcued apoptosis were then investigated.

To date, mitochondrial production of ROS in response to HG may also be a key initiating step in the pathogenesis of DR (29). Our results showed that HG stimulation caused significant increase in the production of ROS in HRECs and the involvement of transcription levels of the oxidative stress associated genes was confirmed. Due to the anti-oxidant and anti-inflammation activities of gastrodin (12), we found treatment of HG exposed HRECs with gastrodin could effectively HG-stimulated oxidative stress. These clarified that gastrodin protected HRECs from HG-induced cell apoptosis via inhibition of oxidative stress related pathway.

It is known that Toll-like receptor 4 (TLR4) signaling pathway participates in the induction of several immune-related 
diseases (30) and is a major contributor of inflammation including TNF- $\alpha$ and IL-1. MyD88 is a common signaling molecule for all TLRs, leading to downstream activation of nuclear factor-kappa beta $(\mathrm{NF}-\kappa \mathrm{B})(31)$. Studies revealed that TLR4 is involved in the activity of late endothelial progenitor cells (32) and was upregulated in the retina of DR rats and in HRECs cultured in HG (33). The TLR4/NF- $\mathrm{B}$ signaling pathway was activated in retinal ganglion cells under high glucose and TLR4 inhibition suppressed HG-induced apoptosis (34). Consist with previous studies, the activation of TLR4/NF- $\kappa \mathrm{B}$ pathway was observed with the treatment of $\mathrm{HG}$ in a dose-dependent manner. Moreover, we identified that SIRT1 may act as a regulator of TLR4/NF- $\kappa \mathrm{B}$ pathway in $\mathrm{HG}$-induced HRECs in vitro. A recent study demonstrated that knockdown of SIRT1 markedly augmented the protein expression of TLR4 and p-NF- $\kappa$ Bp65 in renal inner medullary collecting duct cells treated with lipopolysaccharide (35). In this study, we found SIRT1 protein expression was decreased by HG in a dose-dependent manner and restored SIRT1 significantly caused inhibition of TLR4/NF- $\kappa$ B pathway in HRECs under HG. The role of SIRT1 in diabetic retinopathy had been widely investigated. Hyperglycemia reduced the expression of SIRT1 and restored SIRT1 leading to decreased apoptosis, inflammation, oxidative stress and mitochondrial damage and protects against DR (36). Although a study had identified that SIRT1 could suppress $\mathrm{NF}-\kappa \mathrm{B}$ to reduce inflammatory responses and inhibit cell apoptosis (15), the exact molecular mechanism is still unclear. We firstly determined the key role of TLR4 in regulating NF- $\kappa \mathrm{B}$ signaling under the inhibition of SIRT1 in HG-treated HRECs. We demonstrated that SIRT1/TLR4/NF- $\kappa$ B pathway may be involved in the protective effects of gastrodin on HG-induced. However, the effects and the mechanism of gastrodin on HG-induced impairment of retinal angiogenesis should be further explored in vitro and in vivo.

Additionally, recent research has demonstrated that DR is not only a microvascular disease but may be a result of neurodegenerative processes (37). As the neuroprotective effect of gastrodin had been widely accepted and a recent study found it could also exert a neuroprotective effect on retinal ganglion cells via inhibiting microglia activation and microglial-mediated neuroinflammation (38), we believe gastrodin might be developed as potential candidate for the treatment of DR for both microvascular dysfunction and neurodegeneration.

In summary, this study demonstrated that gastrodin effectively attenuated HG-induced oxidative stress and associated

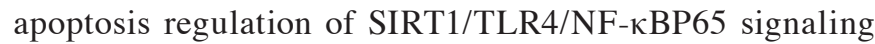
pathway. These findings identified that gastrodin may be an avenue during the treatment of DR.

\section{Acknowledgements}

Not applicable.

\section{Funding}

No funding was received.

\section{Availability of data and materials}

All data generated or analyzed during this study are included in this published article.

\section{Authors' contributions}

QJ designed the study; $\mathrm{CMH}$ and $\mathrm{XG}$ performed the research on cell viability and apoptotic analysis; LLH performed the ROS-associated analysis; and THZ and JW performed the western blot analysis. XG performed statistical analysis, and THZ and JW prepared the manuscript.

\section{Ethics approval and consent to participate}

Not applicable.

\section{Consent for publication}

Not applicable.

\section{Competing interests}

The authors declare that they have no competing interests.

\section{References}

1. Sabanayagam C, Yip W, Ting DS, Tan G and Wong TY: Ten emerging trends in the epidemiology of diabetic retinopathy. Ophthalmic Epidemiol 23: 209-222, 2016.

2. ADVANCE Collaborative Group, Patel A, MacMahon S, Chalmers J, Neal B, Billot L, Woodward M, Marre M, Cooper M, Glasziou P, et al: Intensive blood glucose control and vascular outcomes in patients with type 2 diabetes. N Engl J Med 358: 2560-2572, 2008.

3. Zoungas S, Arima H, Gerstein HC, Holman RR, Woodward M, Reaven P, Hayward RA, Craven T, Coleman RL and Chalmers J; Collaborators on Trials of Lowering Glucose (CONTROL) group: Effects of intensive glucose control on microvascular outcomes in patients with type 2 diabetes: A meta-analysis of individual participant data from randomised controlled trials. Lancet Diabetes Endocrinol 5: 431-437, 2017.

4. Geraldes P, Hiraoka-Yamamoto J, Matsumoto M, Clermont A, Leitges M, Marette A, Aiello LP, Kern TS and King GL: Activation of PKC-delta and SHP-1 by hyperglycemia causes vascular cell apoptosis and diabetic retinopathy. Nat Med 15: 1298-1306, 2009.

5. Roy S, Kern TS, Song B and Stuebe C: Mechanistic insights into pathological changes in the diabetic retina: Implications for targeting diabetic retinopathy. Am J Pathol 187: 9-19, 2017.

6. Kowluru RA, Tang J and Kern TS: Abnormalities of retinal metabolism in diabetes and experimental galactosemia. VII. Effect of long-term administration of antioxidants on the development of retinopathy. Diabetes 50: 1938-1942, 2001.

7. Hou B, He S, Gong Y and Li Z: Effect of obtusifolin administration on retinal capillary cell death and the development of retinopathy in diabetic rats. Cell Biochem Biophys 70: 1655-1661, 2014.

8. Gao J, Zheng Z, Gu Q, Chen X, Liu X and Xu X: Deacetylation of MnSOD by PARP-regulated SIRT3 protects retinal capillary endothelial cells from hyperglycemia-induced damage. Biochem Biophys Res Commun 472: 425-431, 2016.

9. Liu GD, Xu C, Feng L and Wang F: The augmentation of O-GlcNAcylation reduces glyoxal-induced cell injury by attenuating oxidative stress in human retinal microvascular endothelial cells. Int J Mol Med 36: 1019-1027, 2015.

10. Chen J, Gu YT, Xie JJ, Wu CC, Xuan J, Guo WJ, Yan YZ, Chen L, Wu YS, Zhang XL, et al: Gastrodin reduces IL-1 $\beta$-induced apoptosis, inflammation and matrix catabolism in osteoarthritis chondrocytes and attenuates rat cartilage degeneration in vivo. Biomed Pharmacother 97: 642-651, 2018.

11. Zhang Z, Zhou J, Song D, Sun Y, Liao C and Jiang X: Gastrodin protects against LPS-induced acute lung injury by activating Nrf2 signaling pathway. Oncotarget 8: 32147-32156, 2017.

12. Peng Z, Wang S, Chen G, Cai M, Liu R, Deng J, Liu J, Zhang T, Tan $Q$ and Hai C: Gastrodin alleviates cerebral ischemic damage in mice by improving anti-oxidant and anti-inflammation activities and inhibiting apoptosis pathway. Neurochem Res 40: 661-673, 2015. 
13. Jia J, Shi X, Jing X, Li J, Gao J, Liu M, Lin CI, Guo X and Hua Q: BCL6 mediates the effects of Gastrodin on promoting M2-like macrophage polarization and protecting against oxidative stress-induced apoptosis and cell death in macrophages. Biochem Biophys Res Commun 486: 458-464, 2017.

14. Li Q, Niu C, Zhang X and Dong M: Gastrodin and isorhynchophylline synergistically inhibit MPP (+)-induced oxidative stress in SH-SY5Y cells by targeting ERK1/2 and GSK-3 $\beta$ pathways: Involvement of Nrf2 nuclear translocation. ACS Chem Neurosci, 2017 doi: 10.1021/acschemneuro.7b00247.

15. Zhao S, Li T, Li J, Lu Q, Han C, Wang N, Qiu Q, Cao H, Xu X and Chen H: miR-23b-3p induces the cellular metabolic memory of high glucose in diabetic retinopathy through a SIRT1-dependent signalling pathway. Diabetologia 59: 644-654, 2016.

16. Wang L, Wang J, Fang J, Zhou H, Liu X and Su SB: High glucose induces and activates Toll-like receptor 4 in endothelial cells of diabetic retinopathy. Diabetol Metab Syndr 7: 89, 2015.

17. Wang SY, Andrews CA, Herman WH, Gardner TW and Stein JD: Incidence and risk factors for developing diabetic retinopathy among youths with type 1 or Type 2 diabetes throughout the united states. Ophthalmology 124: 424-430, 2017.

18. Luo DW, Zheng Z, Wang H, Fan Y, Chen F, Sun Y, Wang WJ, Sun $\mathrm{T}$ and $\mathrm{Xu} \mathrm{X}$ : UPP mediated diabetic retinopathy via ROS/PARP and NF- $\kappa$ B inflammatory factor pathways. Curr Mol Med 15: 790-799, 2015

19. Shao J, Yin Y, Yin X, Ji L, Xin Y, Zou J and Yao Y: Transthyretin exerts pro-apoptotic effects in human retinal microvascular endothelial cells through a GRP78-dependent pathway in diabetic retinopathy. Cell Physiol Biochem 43: 788-800, 2017.

20. Ojemann LM, Nelson WL, Shin DS, Rowe AO and Buchanan RA Tian ma, an ancient Chinese herb, offers new options for the treatment of epilepsy and other conditions. Epilepsy Behav 8: 376-383, 2006

21. Xu J and Guo S: Retrospect on the research of the cultivation of Gastrodia elata $\mathrm{Bl}$, a rare traditional Chinese medicine. Chin Med J 113: 686-692, 2000.

22. Hua W, Zhu Y and Zhang Q: Determination of gastrodin in human plasma by LC-MS/MS and its application in pharmacokinetic study. Chin J Mod Appl Phar, 2010

23. Cai Z, Huang J, Luo H, Lei X, Yang Z, Mai Y and Liu Z: Role of glucose transporters in the intestinal absorption of gastrodin, a highly water-soluble drug with good oral bioavailability. J Drug Target 21: 574-580, 2013

24. Sun W, Miao B, Wang XC, Duan JH, Ye X, Han WJ, Wang WT, Luo $\mathrm{C}$ and $\mathrm{Hu}$ SJ: Gastrodin inhibits allodynia and hyperalgesia in painful diabetic neuropathy rats by decreasing excitability of nociceptive primary sensory neurons. PLoS One 7: e39647, 2012.

25. Zeng X, Zhang S, Zhang L, Zhang K and Zheng X: A study of the neuroprotective effect of the phenolic glucoside gastrodin during cerebral ischemia in vivo and in vitro. Planta Med 72: 1359-1365, 2006.
26. Luna-Vargas MP and Chipuk JE: Physiological and pharmacological control of BAK, BAX and beyond. Trends Cell Biol 26 906-917, 2016.

27. Vander Heiden MG and Thompson CB: Bcl-2 proteins: Regulators of apoptosis or of mitochondrial homeostasis? Nat Cell Biol 1: E209-E216, 1999.

28. Li M and Qian S: Gastrodin protects neural progenitor cells against amyloid $\beta$ (1-42)-induced neurotoxicity and improves hippocampal neurogenesis in amyloid $\beta$ (1-42)-injected mice. J Mol Neurosci 60: 21-32, 2016.

29. Li J, Yu S, Ying J, Shi T and Wang P: Resveratrol prevents ROS-induced apoptosis in high glucose-treated retinal capillary endothelial cells via the activation of AMPK/Sirt1/PGC-1 $\alpha$ pathway. Oxid Med Cell Longev 2017: 7584691, 2017.

30. Babazada H, Yamashita F and Hashida M: Suppression of experimental arthritis with self-assembling glycol-split heparin nanoparticles via inhibition of TLR4-NF- $\kappa \mathrm{B}$ signaling. J Contro Release 194: 295-300, 2014.

31. Takeda K and Akira S: TLR signaling pathways. Semin Immunol 16: 3-9, 2004.

32. Yu M, Wang C, Zeng G, Zeng G, Zhou L, Chen T, Tan X and Wang Y: Tolllike receptor 4 is expressed and functional in late endothelial progenitor cells. Mol Med Rep 16: 5549-5554, 2017.

33. Berger EA, Carion TW, Jiang Y, Liu L, Chahine A, Walker RJ and Steinle JJ: $\beta$-adrenergic receptor agonist, compound $49 \mathrm{~b}$, inhibits TLR4 signaling pathway in diabetic retina. Immunol Cell Biol 94: 656-661, 2016.

34. Hu L, Yang H, Ai M and Jiang S: Inhibition of TLR4 alleviates the inflammation and apoptosis of retinal ganglion cells in high glucose. Graefes Arch Clin Exp Ophthalmol 255: 2199-2210, 2017.

35. Lin QQ, Geng YW, Jiang ZW and Tian ZJ: SIRT1 regulates lipopolysaccharide-induced CD40 expression in renal medullary collecting duct cells by suppressing the TLR4-NF- $\mathrm{KB}$ signaling pathway. Life Sci 170: 100-107, 2017.

36. Karbasforooshan $\mathrm{H}$ and Karimi G: The role of SIRT1 in diabetic retinopathy. Biomed Pharmacother 97: 190-194, 2018.

37. Zorrilla-Zubilete MA, Yeste A, Quintana FJ, Toiber D, Mostoslavsky R and Silberman DM: Epigenetic control of early neurodegenerative events in diabetic retinopathy by the histone deacetylase SIRT6. J Neurochem 144: 128-138, 2018

38. Wang JW, Liu YM, Zhao XF and Zhang H: Gastrodin protects retinal ganglion cells through inhibiting microglial-mediated neuroinflammation in an acute ocular hypertension model. Int $\mathrm{J}$ Ophthalmol 10: 1483-1489, 2017.

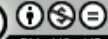

This work is licensed under a Creative Commons Attribution-NonCommercial-NoDerivatives 4.0 International (CC BY-NC-ND 4.0) License. 